\title{
Small-World Network Analysis of Cortical Connectivity in Chronic Fatigue Syndrome Using Quantitative EEG
}

\author{
Mark A. Zinn ${ }^{1 *}$, Marcie L. Zinn ${ }^{1}$, and Leonard A. Jason ${ }^{1}$ \\ ${ }^{1}$ Center for Community Research, DePaul University, Chicago, Illinois, USA
}

\begin{abstract}
The aim of this study was to explore the relationship between complex brain networks in people with Chronic Fatigue Syndrome (CFS) and neurocognitive impairment. Quantitative EEG (qEEG) recordings were taken from 14 people with CFS and 15 healthy controls (HCs) during an eye-closed resting condition. Exact low resolution electromagnetic tomography (eLORETA) was used to estimate cortical sources and perform a functional connectivity analysis. The graph theory approach was used to characterize network representations for each participant and derive the "small-worldness" index, a measure of the overall homeostatic balance between local and long-distance connectedness. Results showed that small-worldness for the delta band was significantly lower for patients with CFS compared to HCs. In addition, delta small-worldness was negatively associated with neurocognitive impairment scores on the DePaul Symptom Questionnaire (DSQ). Finally, delta small-worldness indicated a greater risk of complex brain network inefficiency for the CFS group. These results suggest that CFS pathology may be functionally disruptive to small-world networks. In turn, small-world characteristics might serve as a neurophysiological indicator for confirming a biological basis of cognitive symptoms, treatment outcome, and neurophysiological status of people with CFS.
\end{abstract}

Keywords: chronic fatigue syndrome; myalgic encephalomyelitis; qEEG; eLORETA; electrical neuroimaging; lagged coherence; functional connectivity; graph theory; complex networks; small-world

Citation: Zinn, M. A., Zinn, M. L., \& Jason, L. A. (2017). Small-world network analysis of cortical connectivity in Chronic Fatigue Syndrome using quantitative EEG. NeuroRegulation, 4(3-4), 125-137. http://dx.doi.org/10.15540/nr.4.3-4.125

*Address correspondence to: Mark A. Zinn, Center for Community

Research, DePaul University, 990 W. Fullerton Avenue, Chicago, IL 60614-3504, USA. Email: mzinn@depaul.edu

Copyright: () 2017. Zinn et al. This is an Open Access article distributed under the terms of the Creative Commons Attribution License (CC-BY).

\author{
Edited by: \\ Rex L. Cannon, PhD, Knoxville Neurofeedback Group, Knoxville, \\ Tennessee, USA

\section{Reviewed by:} \\ Wesley D. Center, PhD, Liberty University, Lynchburg, Virginia, USA \\ Randall Lyle, PhD, Mount Mercy University, Cedar Rapids, lowa, \\ USA
}

Chronic fatigue syndrome (CFS) is a complex multisystem disease characterized by unexplained persistent or relapsing fatigue, post-exertional malaise, flu-like symptoms, and neurocognitive impairments not relieved by rest and worsened by physical or mental activity (Carruthers et al., 2003; Fukuda et al., 1994). Neurocognitive impairment is a hallmark symptom in CFS (Jason, Zinn, \& Zinn, 2015) and one of the primary factors involved in the etiology of the condition (Johnson, DeLuca, \& Natelson, 1996). Approximately $90 \%$ of patients with CFS report having cognitive symptoms, anecdotally referred to in the clinic as "brain fog," profoundly affecting health and quality of life (Grafman et al., 1993; Hopkins \& Jackson, 2006;
Komaroff \& Buchwald, 1991; Ocon, 2013). A metaanalysis found cognitive deficits in CFS pertaining to memory, attention, and information processing speed, particularly during sustained working memory tasks (Cockshell \& Mathias, 2010). In addition, patients have been shown to have slower reaction times in many studies (Busichio, Tiersky, DeLuca, \& Natelson, 2004; Constant et al., 2011; Majer et al., 2008; Thomas \& Smith, 2009; Van Den Eede et al., 2011), particularly under conditions of increasing task complexity (Dobbs, Dobbs, \& Kiss, 2001). DeLuca, Johnson, and Natelson (1994) proposed that most memory deficits seen in patients are due to slower information processing rather than impairment in storage/retrieval mechanisms. 
Functional MRI studies have observed that patients with CFS show signs of brain compensation in response to verbal working memory tasks (Cook, O'Connor, Lange, \& Steffener, 2007; Lange et al., 2005). This suggests that dynamic reorganization of brain network topology in CFS with subsequent reductions in neural efficiency could be contributing to cognitive impairment indirectly. Thus, examining changes in overall brain information processing speed and neural efficiency factors in CFS may elucidate the relationship between cortical dysregulation and cognitive symptoms.

Knowledge of general principles of self-organization in real-word systems has prompted a paradigm shift in neuroscience away from localization of brain responses toward a deeper understanding of brain connectivity influences on information processing efficiency (Sporns, 2013). In the past decade, graph theoretical analysis has been increasingly used in neuroscience as a framework for understanding how dynamic processes are involved in the emergence of cognition and behavior (Menon, 2012; Stam, 2014). This approach has a number of distinct advantages which allow researchers to: 1) quantify and model a wide range of varying network attributes, 2) characterize the balance of local and global tradeoffs that operate within systems, 3) examine weakened elements of the system and compensatory dynamics responding to pathological processes, and 4) simultaneously account for relationships between all the network elements and a given cognitive function (Rubinov \& Sporns, 2010). In this sense, the application of graph theoretical analysis can extend our understanding of the key aspects of brain function in patients with CFS.

Complex networks are ubiquitous to the real world (e.g., social networks, airline routes, power grids, protein networks; Watts \& Strogatz, 1998), and the brain itself is a complex network comprised of many subnetworks of distributed brain regions which instigate even the most basic behaviors (Deco, Jirsa, \& Friston, 2012; Sepulcre, 2014; Stam, 2010). The coordinated activity within complex networks of the brain gives rise to fundamental aspects of neurocognitive domains involving attention, perception, memory, language, and motor processing (van den Heuvel \& Sporns, 2013; Wig, Schlaggar, \& Petersen, 2011). A homeostatic balance exists within complex brain networks between random neuronal growth processes and activity-dependent modification of those processes (Minati, Varotto, D'Incerti, Panzica, \& Chan, 2013). This state of affairs can be explained in terms of parsimony; there is a continual drive in the system to negotiate trade-offs to the costs involved in supporting and to create adaptively valuable functional connectivity (Bullmore \& Sporns, 2012). The number of connections in the system is relegated by wiring cost (biological energy and materials), and there are evolutionary reasons for keeping the demand for long distance connections, which are more "expensive," to a minimum (Stam, 2010). Peculiar trade-offs in the topological properties of complex brain networks can therefore serve as a marker for specific neurobiological adaptions to the CFS condition, modeling disease course and spread, aberrant plasticity, indexing overall information processing efficiency-all of which could aid clinical diagnosis of patients and even identify clinical subtypes (Crossley et al., 2014).

The "small-world" network model was introduced in a landmark study by Watts and Strogatz (1998) demonstrating for the first time that small-world properties exist in central nervous systems. The topology of small-world networks is characterized by high clustering (segregation) and short path lengths (integration), representing a homeostatic balance between local and global processing in order to satisfy opposing demands which maximize processing speed at minimal neurobiological energy cost (Sporns \& Honey, 2006). Segregation refers to the tendency of nearest neighbor elements to cluster together, whereas integration refers to the amount of interconnectedness and efficient information exchange within the entire network. The clustering coefficient is a measure of functional segregation or local connectedness, whereas the characteristic path length is a measure of functional integration describing global, large-scale activity and coactivation of neuronal populations within the network (Telesford, Simpson, Burdette, Hayasaka, \& Laurienti, 2011). The clustering coefficient and the characteristic path length constitute properties of the small-world network model. Taken together, they are an indicator of small-worldness, an index representing the suitable balance between functional integration and segregation of dynamic system organization (Humphries \& Gurney, 2008; Stam, 2010; Thatcher, 2016; van Straaten \& Stam, 2013).

Kim et al. (2015) demonstrated small-world abnormality in CFS using resting-state fMRI to examine a sample of 18 women with CFS and 18 age-matched female controls. They assessed global efficiency, the inverse of the mean shortest characteristic path length, relating to the functional efficiency of information flow between any two nodes in the network. They also assessed local efficiency 
which quantifies the fault tolerance of the network proportional to the clustering coefficient (Bassett \& Bullmore, 2006). They found that global efficiency (integration) was lower in CFS compared to the HC group, while there were no differences in local efficiency (segregation). Increased demand for long distance connections in CFS suggests there is an added wiring expense for compensatory systems which negatively affects global efficiency of the network information processing. The degree of perturbation to small-world dynamics was linked to the amount of neurocognitive impairment in patients and brain processes found to be compromised reflected an underlying disturbance to small-world propensity. However, these investigators did not examine small-worldness, an overall indicator of optimal brain functioning and neural efficiency and neurocognitive impairment (e.g., memory, attention, slow thought, etc.) may represent a combination of pathology in the overall small-worldness measure with the concomitant overt behavioral changes in CFS.

Quantitative electroencephalography (qEEG) involves numeric analysis of local field potentials resulting from the summation of neuronal electrical activity that arises from the cell bodies and associated dendrites of large populations of synchronously active cortical pyramidal neurons (Niedermeyer \& Lopes da Silva, 2005). The electrical currents are dependent on the integrity of the neural sodium/potassium and calcium ion pumps, reflecting metabolic activity and rendering qEEG a useful tool for quantifying and exploring electrophysiological correlates of both normal and abnormal neurological function (Thatcher, 2016). The frequency, phase, and amplitude of band-limited EEG oscillations relates to the specific information processing taking place at different spatiotemporal scales at any given moment (Le Van Quyen, 2011). Higher order cognitive processes appear to call upon even more temporal precision for sustained neuronal activity between neuronal populations (Nunez, Srinivasan, \& Fields, 2015). Temporal resolution of qEEG on a millisecond timescale allows fine-grained detection of subtle differences in speed and efficiency within the relay of information flow via cooperative sequencing of oscillatory patterns and their phase differences (Buzsáki \& Freeman, 2015; Steriade, 2005; Thatcher, North, \& Biver, 2008). This is important given that even the most basic cognitive processes depend on precise timing of phase relationships in the brain occurring through large populations of spontaneously synchronized neurons communicating among distributed brain regions (Buzsáki, 2006; Sauseng \& Klimesch, 2008; Steriade \& Paré, 2006).

Tomographic EEG methods (electrical neuroimaging) use inverse methods to accurately map current source density in a three-diminensional brain volume, allowing the ability to visualize EEG abnormality in deeper brain structures (Grech et al., 2008; Thatcher, 2016). A growing number of studies are using electrical neuroimaging methods to elucidate information processing in the brain and small-world network organization in response to neurological conditions including epilepsy (Adebimpe, Aarabi, Bourel-Ponchel, Mahmoudzadeh, \& Wallois, 2016; Vecchio, Miraglia, Curcio, Della Marca, et al., 2015), multiple sclerosis (Vecchio et al., 2017), and Alzheimer's disease (Hata et al., 2016; Vecchio, Miraglia, Curcio, Altavilla, et al., 2015; Vecchio et al., 2016). A comprehensive review on the role of electrical neuroimaging techniques for studying the brain in CFS can be found in Jason, Zinn, et al. (2015).

Using low resolution electromagnetic tomography (LORETA) to investigate 17 monozygotic twins with one twin with CFS vs. one healthy co-twin, Sherlin et al. (2007) showed that twins affected with CFS had increased delta sources in the left uncus and parahippocampal gyrus, deeper structures of the limbic system. Sherlin et al. also found higher theta sources in the cingulate gyrus and right superiorfrontal gyrus. Using eLORETA (where "e" stands for exact), Zinn et al. (2014) found significantly elevated delta sources in a widespread portion of the frontal lobe and limbic lobe as well as decreased beta sources in the parietal lobe bilaterally. Higher delta sources were also associated with the reduced motivation scores on the Multidimensional Fatigue Inventory, a measure of fatigue severity commonly used in CFS studies. Increased delta in limbic structures is consistent with the findings of Sherlin et al., and rhythmic alterations in these regions could be indicators of blunted emotional processing in CFS possibly related to reduced motivation and attentional difficulties. Interestingly, symptoms manifested by brain pathology within the medial prefrontal cortex, anterior cingulate, and orbitofrontal cortex are largely undetected by most traditional neuropsychological tests (Koziol \& Budding, 2009). Finally, using a Beamformer source analysis method, Flor-Henry, Lind, and Koles (2010) found sources that were globally reduced in the alpha and beta bands in those with CFS (delta band was not examined). Together, the various $\mathrm{qEEG}$ and tomographic EEG investigations mentioned here 
demonstrate a relationship between EEG and CFS which lay the foundations for this study.

Zinn, Zinn, and Jason (2016) performed eLORETA functional connectivity analysis in CFS to examine three fundamental neurocognitive networks based on Menon's triple network model of brain pathology (Menon, 2011). This model posits there are three primary large-scale brain networks that operate dynamically to regulate shifts in arousal, attention, and general access to cognitive abilities. It includes the central executive network, salience network, and the default mode network and predicts that aberrant activity within any one of these networks will significantly impact the other two networks resulting in pathological states. Using lagged phase synchronization (Pascual-Marqui, 2007a), hypoconnectivity was found in the delta and alpha frequency bands between nodes for all three networks in the group with CFS in comparison to health controls. This finding is consistent with several functional connectivity studies using magnetic resonance which reported decreased connectivity involving key nodes of the salience network (Boissoneault et al., 2016; Gay et al., 2016; Wortinger et al., 2016). Disruptions to the salience network could underlie primary cognitive symptoms in CFS involving attention to internal/external events and adaptive engagement of systems responsible for processing of working memory and executive control. The above findings show that functional connectivity approaches including electrical neuroimaging methods are promising avenues for studying brain dysfunction in CFS.

The present study addressed the question of whether fundamental neurobiological relationships and adaptions could underlie cognitive symptoms in CFS. Our primary hypothesis was that patient networks would show deviations from normal in small-world network characteristics as measured by the small-worldness index, thus demonstrating a pathological imbalance affecting network efficiency and information processing due to the trade-offs associated with adaptive reconfiguration of network topology in CFS. Using graph theoretical analysis of small-world networks with eLORETA connectivity data was used for exploring the linkage of brain topology with cognitive impairments that are commonly associated with CFS (John, 2005; van Straaten \& Stam, 2013). Secondly, changes in the small-worldness index were hypothesized to be associated with subjective levels of cognitive impairment due to maladaptive reconfigurations in network topology needed for supporting efficient brain processing in patients with CFS. Lastly, the small-worldness index was tested as a way to look at risk in patients with CFS compared to $\mathrm{HC}$ participants. At the present time, there is no physiological marker that represents risk for neurocognitive impairment in patients with CFS. Having an accurate method for identifying risk of cognitive impairment in CFS would help establish the utility for this approach for identifying epidemiological factors relating to patient health.

\section{Method}

\section{Participants}

The participants in this investigation were 29 adults (14 individuals with CFS, $15 \mathrm{HCs}$ ) ranging in age from 20 to 80 years old and the mean age was 43.97 years $(S D=20.32)$. The effects of age were statistically adjusted since the mean age between groups was significantly different and physiological aging is a significant factor within the EEG (Kirk, 2013; Rossini, Rossi, Babiloni, \& Polich, 2007; Vysata et al., 2014). All participants visited the Center for Community Research at DePaul University to have their EEG recorded. The participants with CFS all met the Fukuda criteria (Fukuda et al., 1994) and they had been diagnosed with CFS by their physician. No participants were taking medications that would affect the EEG. This study was approved by the Institutional Review Board at DePaul University in Chicago.

\section{Procedure}

Eyes-closed, resting state EEG data for each participant was recorded for 5 min from 19 electrode locations (Fp1, Fp2, F3, F4, F7, F8, Fz, C3, C4, Cz, P3, P4, Pz, T3, T4, T5, T6, O1, and O2) positioned on the scalp according to the international 10/20 system using standardized electrode caps (Jurcak, Tsuzuki, \& Dan, 2007) with a linked-ears reference. During cap preparation, impedances for all electrode sites were measured and brought to within $5 \mathrm{k} \Omega$. Once cap preparation was completed, participants were shown their raw EEG signals and trained to minimize artifact by relaxing muscles in their forehead, jaws, and face to the best of their ability while they observed corresponding changes in the raw EEG. The data collection apparatus involved Neuroguide qEEG signal processing software (Version 2.8.7, 2016) together with the BrainMaster Discovery 24 (Bedford, OH) qEEG acquisition module, which allows up to 19 channels of EEG signals to be recorded simultaneously at $256 \mathrm{~Hz}$.

During the EEG recording session, each participant was seated upright in a comfortable chair in a room that was well lit. Participants were given instructions to relax to the best of their ability while keeping their 
eyes closed until the recording session has ended. EEG data were acquired at a $256 \mathrm{~Hz}$ sampling rate and filtered offline between 1 and $40 \mathrm{~Hz}$. Artifact removal procedures were as follows: 1) visual inspection and manual deletion of visible artifact by an EEG technician; 2) automated Z-score artifact removal using rejection algorithms built into Neuroguide set for high sensitivity at two standard deviations for immediate exclusion of EEG segments with eye movement, muscle, and drowsiness artifact; and 3 ) second visual inspection and manual deletion of the artifact by an EEG technician. Since this study was directed toward understanding changes in phase relationships of the original time-series data, independent components analysis (ICA) was not performed. ICA/regression procedures intended to remove artifact actually produce distortion of phase relationships between channels by reconstructing the EEG time series. This methodological problem, which essentially invalidates the EEG data, has been empirically proven in several studies (Castellanos \& Makarov, 2006; Kierkels, van Boxtel, \& Vogten, 2006; Wallstrom, Kass, Miller, Cohn, \& Fox, 2004). The EEG segments that were included for analysis showed greater than $95 \%$ split-half reliability and greater than $90 \%$ test-retest reliability coefficients instantaneously computed by Neuroguide, and each record had a minimum total edit time of at least 1 minute. For each participant, the artifact-free data were then fragmented into 2sec EEG segments. Due to theoretical considerations, all analyses were limited to the delta (1-3 Hz), alpha-1 (8-10 Hz), and alpha-2 (10-12 $\mathrm{Hz}$ ) frequency bands. Each frequency band provides an added layer of physiological significance to brain function.

\section{Materials}

All participants completed the DePaul Symptom Questionnaire (DSQ; Jason, So, Brown, Sunnquist, \& Evans, 2015), and data for the DSQ were collected and managed using the Research Electronic Data Capture (REDCap) hosted at DePaul University (Harris et al., 2009). The DSQ is a self-report instrument that measures 54 symptoms related to criteria specified in the CDC criteria (Fukuda et al., 1994), the Canadian Criteria for ME/CFS (Carruthers et al., 2003), and the CFS International Consensus Criteria (Carruthers et al., 2011). For each symptom item, respondents are asked to separately rate the frequency and severity over the last 6 months on a 5-point Likert scale $(0=$ none of the time, $1=$ a little of the time, $2=$ about half the time, $3=$ most of the time, and $4=$ all of the time). The DSQ has good test-retest reliability with Pearson's correlation coefficients above 0.70 and test-retest correlations for classified symptom categories (fatigue, post-exertional malaise, neurocognitive, and autonomic) at 0.80 or higher (Jason, So, et al., 2015). Results of factor analysis on the DSQ support at least three distinct symptom factors: 1) post-exertional malaise, 2) neurocognitive dysfunction, and 3) neuroendocrine/autonomic limmune dysfunction (Jason, Sunnquist, et al., 2015). Murdock et al. (2016), an independent group using the DSQ, found that it demonstrated excellent internal reliability and that among patient-reported symptom measures it optimally differentiated between patients and controls. The cognitive variable of this proposal was the aggregate average of nine items that fall under the neurocognitive dysfunction factor: problems remembering things, difficulty paying attention for a long period of time, difficulty with word finding or expressing thoughts, difficulty understanding things, only able to focus on one thing at a time, unable to focus vision attention, slowness of thought, absentmindedness or forgetfulness, and loss of depth perception (Jason, Sunnquist, et al., 2015).

Functional connectivity was analyzed using coherence, a measure of the consistency of phase differences in the time-series corresponding to different spatial locations (Lehmann, Faber, Gianotti, Kochi, \& Pascual-Marqui, 2006; Pascual-Marqui, 2007a, 2007b). Coherence is interpreted as an indicator of "connectivity" which quantifies the degree to which phase differences remain stable over time either between electrode sites, when measured at the scalp when using surface EEG (Buzsáki \& Watson, 2012; Klimesch, Freunberger, Sauseng, \& Gruber, 2008; Thatcher, 2016), or between two brain regions, in the case of eLORETA (Pascual-Marqui et al., 2011). An advantage of eLORETA is that it uses lagged coherence, a specialized measure of functional connectivity that controls for physiological artifact by removing zerolag contributions from volume conduction and spatial blurring effects (Pascual-Marqui, 2007a, 2007c). Functional connectivity analyses of coherence was conducted using the LORETA-KEY software package (Pascual-Marqui, 2015). This software is freely provided for download by the KEY Institute for Brain-Mind Research at http://www.uzh.ch/keyinst /loreta.htm. eLORETA is based on the stereotactic space provided by the Montreal Neurological Institute (MNI) template and offers a highly accurate estimate of the intracortical current source density within a three-dimensional cortical volume consisting of 6,239 voxels of unambiguous grey matter at 5 $\mathrm{mm}^{3}$ spatial resolution. Complete mathematical details of this inverse solution are provided in 
Pascual-Marqui et al. (2011). To obtain a topographic view of the whole cortex, coordinates were computed for 42 separate Brodmann areas (BAs: 1, 2, 3, 4, 5, 6, 7, 8, 9, 10, 11, 13, 17, 18, 19 $20,21,22,23,24,25,27,28,29,30,31,32,33,34$, $35,36,37,38,39,40,41,42,43,44,45,46,47)$ for the left and right hemispheres (84 total ROIs) using a single voxel to define each ROI centroid. Given that eLORETA has low spatial resolution based on the spatial smoothness assumption, the single center voxel is considered an accurate representation of activity within the ROI while minimizing the possibility of signal contamination from neighboring ROIs.
eLORETA lagged coherence was then calculated for all 84 ROls for each participant, generating text files with output containing a separate weighted $84 \times 84$ coherence matrix for each frequency band. The coherence matrix contains the entire set of network connections whereby each cell has a value representing the magnitude of the statistical correlation (coherence) between any pair of nodes. In each coherence matrix, the table rows and columns represent the ROls and the cell values represent the coherence magnitude of dependency between each pair of ROls. Figure 1 illustrates the workflow for all the analyses that were implemented in this study.
A. Surface EEG data

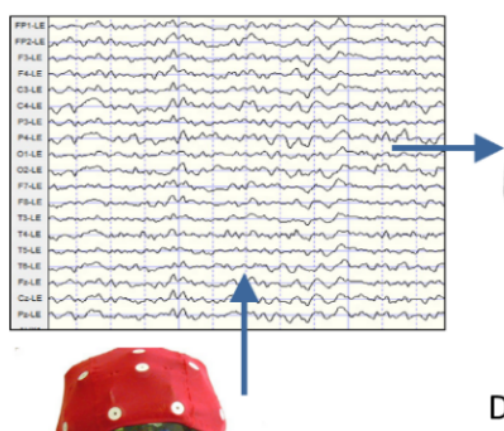

D. Graph analysis

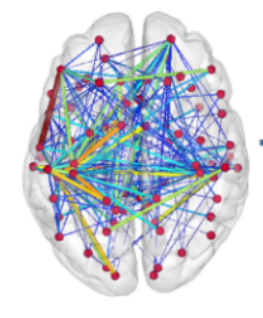

B. Define ROIs

\section{Obtain coherence matrices per subject}

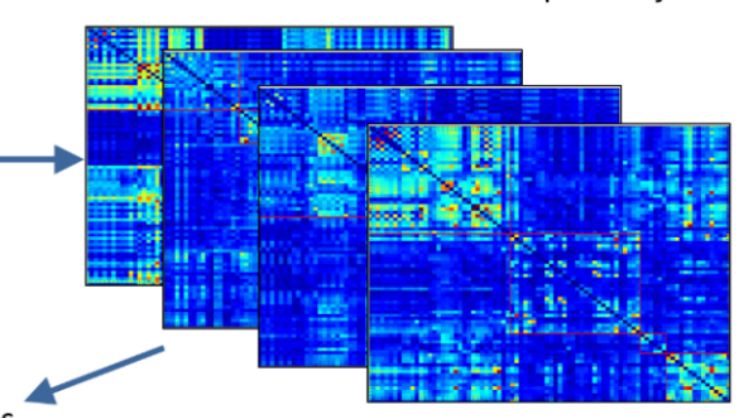

E. Statistical testing

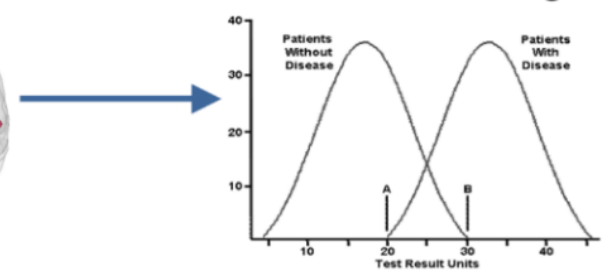

Figure 1. The workflow of all analyses in this thesis summarized as an overview.

\section{Graph Theoretical Analysis}

The coherence matrix for each frequency band for each participant was subjected to graph theoretical analysis using the MATLAB Brain Connectivity Toolbox (BCT; Rubinov \& Sporns, 2010). The BCT has functions that take into account the weighted undirected strength or magnitude of all the network connections. Descriptions and code for the mathematical functions in the BCT are freely available for download at https://sites.google.com /site/bctnet/. BCT functions were applied to each participant's coherence matrix to calculate smallworld characteristics. The weighted clustering coefficient around a given node varies from 0 to 1 and is quantified by the number of triangles formed by that node and its neighboring nodes. The weighted characteristic path length is defined as the average shortest weighted path between two given nodes using the sum of the individual weighted lengths. Path lengths with conversions based on values of the coherence matrix were stored separately as a distance matrix with sequences of edges that connect nodes indirectly to form neural paths. The path length values in the distance matrix are not physical distances, but instead they represent the degree of topological separation between any two given nodes (Rubinov \& Sporns, 2010). The GraphVar toolbox in Matlab (Kruschwitz, 
List, Waller, Rubinov, \& Walter, 2015) was used to calculate small-worldness, which is the ratio between the clustering coefficient and characteristic path length compared to their values for equivalent randomly generated graphs (Humphries \& Gurney, 2008). The small-worldness index variable (SW) was computed using $C_{\mathrm{sw}}=\left(C w / C w_{\text {rand }}\right) /\left(L w / L w_{\text {rand }}\right)$ as a comparative marker of efficient brain functioning for each participant.

\section{Statistical Analysis}

The graph theory output that was produced using $B C T$ functions in MATLAB was subsequently imported to SPSS version 23 for conducting further statistical analyses. The data were screened for outliers, missing data, skewness, and kurtosis in meeting the assumptions for parametric statistics.
Continuous variables were log-transformed to meet the assumption of Gaussianity.

\section{Results}

Demographic characteristics by study group and descriptives of key study variables are shown in Tables 1 and 2. Most patients with CFS were older than $\mathrm{HCs}$ and the potential confound of age was controlled for in all models. Given that some secondary outcomes were considered corresponding to the study hypotheses, this study is considered exploratory, and the $p$ values considered descriptive. All data were evaluated with tests which were two-sided at the .05 level of significance.

\begin{tabular}{|c|c|c|c|c|c|c|}
\hline & & 14 CFS & & $15 \mathrm{HCs}$ & & 29 Participants \\
\hline $\begin{array}{l}\text { Age }(\text { years })^{\star} \\
\text { Mean }(S D)\end{array}$ & & $57.71(15.15)$ & & $31.13(15.63)$ & & $43.97(20.32)$ \\
\hline \multirow[t]{2}{*}{ Sex } & 11 & Female & 11 & Female & 22 & Female \\
\hline & 3 & Male & 4 & Male & 7 & Male \\
\hline \multirow[t]{2}{*}{ Handedness } & 14 & Right & 14 & Right & 28 & Right \\
\hline & 0 & Left & 1 & Left & 1 & Left \\
\hline \multirow[t]{3}{*}{ Education } & 1 & Partial college & 2 & Partial college & 3 & Partial college \\
\hline & 6 & College & 8 & College & 14 & College \\
\hline & 7 & Graduate & 5 & Graduate & 12 & Graduate \\
\hline \multirow[t]{2}{*}{ Ethnicity } & 14 & White & 14 & White & 28 & White \\
\hline & & & 1 & Latino & 1 & Latino \\
\hline $\begin{array}{l}\text { DSQ Cognitive Composite score* } \\
\text { Mean }(S D)\end{array}$ & & $2.87(.10)$ & & $1.25(.04)$ & & $2.03(.10)$ \\
\hline
\end{tabular}

${ }^{*} p<.01$

\section{Table 2}

Means and Confidence Intervals for Small-worldness Indices by Experimental Group.

\begin{tabular}{ccccc}
\hline & & $\begin{array}{c}\text { Small-world } \\
\text { delta }\end{array}$ & $\begin{array}{c}\text { Small-world } \\
\text { alpha-1 }\end{array}$ & $\begin{array}{c}\text { Small-world } \\
\text { alpha-2 }\end{array}$ \\
\cline { 3 - 5 } Group & $N$ & $M(C l)$ & $M(C l)$ & $M(C l)$ \\
\hline HC & 15 & $.89(.85-.92)$ & $.79(.76-.83)$ & $.84(.80-.88)$ \\
CFS & 14 & $.82(.78-.85)$ & $.78(.74-.82)$ & $.79(.76-.82)$ \\
\hline
\end{tabular}

The primary outcome of interest was to determine whether small-world network values deviate from normal in a sample of patients with CFS. Analysis of
Variance (ANOVA) was conducted to assess whether networks of patients with CFS deviated significantly from those of $\mathrm{HCs}$, adjusting for age. We first identified statistically significant ANOVA values in an overall test, $F(2,80)=4.915, p=.029$, which indicated a significantly lower small-worldness index $z$-value for patients with CFS $(M=-.181, S D=$ 1.047) than HCs $(M=.164, S D=.950)$. To identify the differences between small-worldness within each frequency band in this study, follow-up tests were conducted with the Bonferroni correction for multiple comparisons. These estimates identified SW delta as statistically different between patients with CFS and HCs, $p=.014$; however, the SW alpha-1 and 
SW alpha-2 were not significant between both groups ( $p=.622$ for alpha-1; $p=.099$ for alpha-2; Figure 2). Within the HC group, a significant difference was found between SW delta and SW alpha-1 $(p=.001)$, but not between SW delta and SW alpha-2 $(p=.177)$. Within the CFS group, however, there was no significant difference between any SW frequency bands $(p=.355)$.

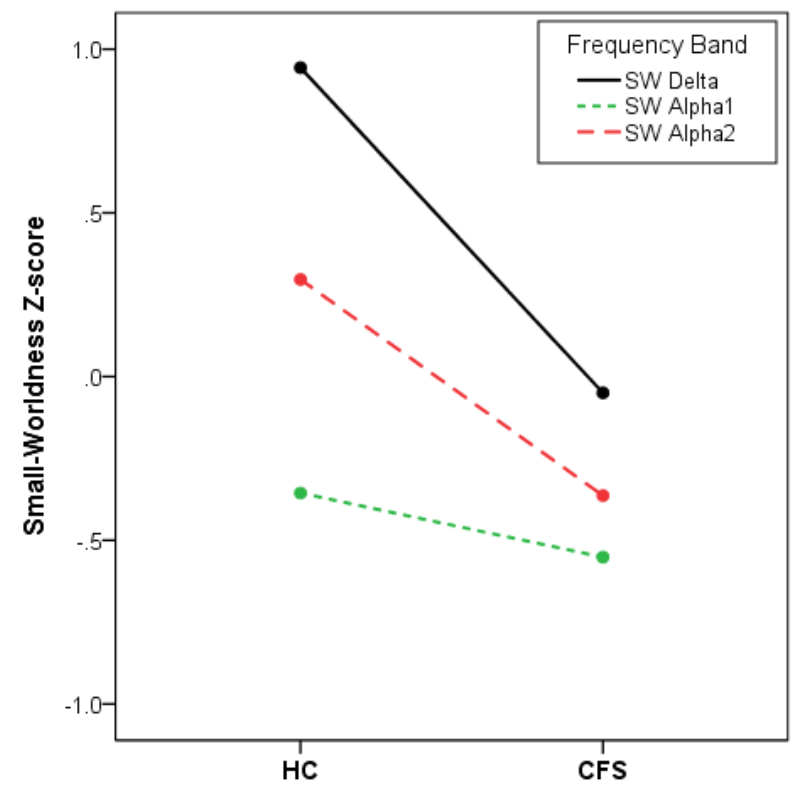

Figure 2. Small-worldness results of group comparisons by frequency band. The CFS group was 1 SD lower than the HC group for SW delta $(p=.014)$.

Next, hierarchical regression techniques were used to determine the linear relationship of small-world network organization (measured by SW delta, SW alpha-1, and SW alpha-2 combined) with neurocognitive impairment. Two models were fit for estimating this relationship, age-adjusted, and found that small-worldness significantly predicted the neurocognitive impairment scores, $F(2,84)=$ 53.482, $p=.000$, adjusted $R^{2}=.550$ for model 1 and $F(2,83)=122.546, p=.000$, adjusted $R^{2}=.809$. These strong effect sizes suggest that deviations from small-worldness affect neurocognitive impairment. For model 2 in particular, $80.9 \%$ of neurocognitive impairment was predicted by the combination of small-worldness, experimental group, and age (Figure 3).

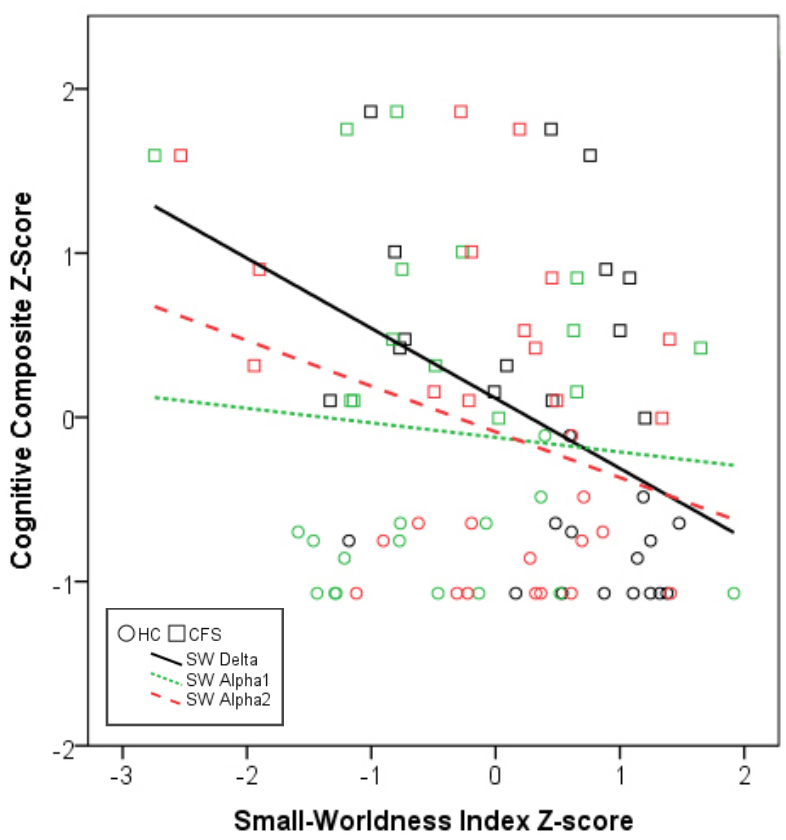

Figure 3. Small-worldness results of regression analysis by frequency band.

Our third outcome of interest was the development of prediction models to estimate odds ratios and $95 \%$ Cls for patients with CFS in SW delta, SW alpha-1, and SW alpha-2. Fixed-effects multinomial logistic regression allowed us to appropriately model the relationship between group membership and small-world effects at each frequency band. All models were adjusted for the potential confounder of age. To estimate differences between patients with CFS in our study cohort, the deviated small-world values (small-worldness index variable) in the fixedeffects logistic regression model were associated with increased risk in CFS of SW delta (OR 1.425; 95\% Cl: $0.500-3.75$ ) but not for SW alpha-1 (OR $0.702 ; 95 \% \mathrm{Cl}: 0.310-1.590$ ) or SW alpha-2 (OR $0.786 ; 95 \% \mathrm{Cl}: 0.386-1.601)$. According to this data set, the group with CFS was 1.425 times as likely to have deviations from normal in small-worldness in the delta frequency band but not in the alpha- 1 or alpha-2 band. The overall regression model was significant at $p=.05$.

\section{Discussion}

To our knowledge, this is the first study to evaluate an association between small-world characteristics and cognitive symptoms reported in CFS. These findings of functional connectivity alterations suggest the importance of applying graph theory to connectome-scale analysis of network topology to detect subtle disruptions incurred by CFS sequelae. 
Neurocognitive impairment, as measured by the DSQ cognitive composite score, was negatively associated with small-worldness index for the delta band under observation. Group-level differences were also found, but only for small-worldness in the delta band. Finally, the risk of having smallworldness deviations in the delta band is increasingly greater in CFS.

Small-world models of the brain systems explore the balance between high clustering of local systems with short path lengths of global systems; these attributes are considered to be vital to the efficiency of information processing within neurocognitive networks (Menon, 2012; Rubinov \& Sporns, 2010). This model emphasizes the morphological adaptations (e.g., changes in axonal diameter, white matter pathways, conduction velocities, and energy transport mechanisms) governed by trade-offs within components and compensation necessary for maintaining the multiscale spatial-temporal patterns for which the brain operates. Differences in neural resource allocation in CFS were reported in three fMRI studies investigating compensatory responses to cognitive tasks (Caseras et al., 2006; Cook et al., 2007; Lange et al., 2005). The findings of our study explain these differences in terms of peculiarities to these trade-offs with subsequent weakness to smallworldness structure that could account for loss of cognitive function in people with CFS.

Secondarily, it was found that small-worldness in the delta band accounted for the greatest amount of variance in cognitive composite scores for the hierarchical regression model equation. Delta is a slow oscillation that plays a key role in the dynamic coordination of large-scale cortical networks and modulation of faster rhythms through crossfrequency coupling (Buzsáki \& Freeman, 2015). In the case of inflammatory disorders of the CNS, the most prominent change in large-scale network dynamics is the occurrence of cortical slowing (e.g., delta activity) during the waking state (Westmoreland, 2005). Furthermore, delta cortical slowing can result from a decrease in the afferent drive due to white matter or subcortical lesions to deep midline areas (Gloor, Ball, \& Schaul, 1977; Schaul, Gloor, \& Gotman, 1981). Finding abnormal small-worldness in delta suggests there may be some similarities between CFS and Alzheimer's disease (Babiloni et al., 2013; Hata et al., 2016), multiple sclerosis (Babiloni et al., 2016), and Parkinson's disease (Babiloni et al., 2011), where abnormal delta sources have been detected.
This is the first study to measure small-world properties in CFS in terms of the small-worldness index. Using resting-state fMRI data, Kim et al. (2015) found that functional integration (global efficiency) was decreased in CFS and disruption to global efficiency suggests that, with fewer biologically "expensive" long distance connections, added burden is being placed on the system for satisfying opposing demands. The "costs" to chronically reduced functional integration in CFS include: 1) a lowered ability to rapidly combine specialized information from distributed brain regions, 2) slowed information processing speed due to compensatory responses, and 3) a generalized impairment to domains of cognitive function. However, our study found differences using the small-worldness index as a ratio of individual small-world properties (clustering and path lengths), a measure of both global and local properties which are salient in CFS depending on frequency band. This underscores the need for considering a combination of graph theory metrics for a more comprehensive examination of CFS.

There are some limitations in the present study. The results of this study should be interpreted with caution due to small sample size. Although significant deviations in the reported smallworldness phenomena were found in people with CFS, neurological disorders are invariably associated with diffuse network changes. However, it was beyond the scope of this study to report the individual nodes, hub, and modules that may be involved in suboptimal information processing efficiency and prone to failure in CFS. Although the outcome of brain function following individual hub failure would likely go beyond discrete local regions, future research could explore a more comprehensive inspection of hub strength, distribution, and participation within modular structures to identify ROIs that serve as potential targets for treatment. As another limitation of this study, the examination of small-world differences was kept within the delta, apha-1, and alpha-2 frequency bands. Frequency-dependent changes to cortical arrangements occurring in other frequency bands (e.g., theta, beta) could also be explored. Finally, insignificant findings in alpha-1 and alpha-2 could reflect a deficiency in the diagnostic criteria for CFS, a deficiency in the coherence-based measure itself, a problem with the way the ROls were defined, and/or unexplored levels of complex network analysis using other graph theory metrics. Functional connectivity EEG markers associated with neurocognitive impairment and small-worldness 
in different frequency bands should be verified in future studies.

\section{Conclusions}

The present findings support the concept that smallworldness is altered in CFS. This has important implications for this field of study. For example, system-dependent coupling of oscillations has fundamental importance to CNS function and may be strongly influenced by delays in conduction velocity and myelin plasticity. Changes to white matter have been reported in CFS (Puri et al., 2012), also associated with clinical measures (Barnden et al., 2011), and a severity-dependent increase in myelination has also been found (Barnden, Crouch, Kwiatek, Burnet, \& Del Fante, 2015). Disruption to white matter could explain the relationship between abnormal eLORETA coherence patterns over largescale complex systems in CFS. Furthermore, the linkage between cognitive symptoms and smallworldness demonstrates the fundamental importance of timing, stability, and adaptation of complex systems to CFS which could be related to findings of neuroinflammation in patients (Nakatomi et al., 2014). Understanding the network dynamics of CFS in terms of eLORETA coherence is an important way of comprehending compensatory mechanisms and could serve as a practical tool for investigating large-scale loss of cognitive function related to adaptive reconfiguration of brain networks. There is a need for future research that models the activity-dependent modifications of brain connectivity in CFS with disruption to neurocognitive processes.

\section{References}

Adebimpe, A., Aarabi, A., Bourel-Ponchel, E., Mahmoudzadeh, M., \& Wallois, F. (2016). EEG resting state functional connectivity analysis in children with benign epilepsy with centrotemporal spikes. Frontiers in Neuroscience, 10, 143. http://dx.doi.org/10.3389/fnins.2016.00143

Babiloni, C., Carducci, F., Lizio, R., Vecchio, F., Baglieri, A., Bernardini, S., ... Frisoni, G. B. (2013). Resting state cortical electroencephalographic rhythms are related to gray matter volume in subjects with mild cognitive impairment and Alzheimer's disease. Human Brain Mapping, 34(6), 14271446. http://dx.doi.org/10.1002/hbm.22005

Babiloni, C., De Pandis, M. F., Vecchio, F., Buffo, P., Sorpresi, F., Frisoni, G. B., \& Rossini, P. M. (2011). Cortical sources of resting state electroencephalographic rhythms in Parkinson's disease related dementia and Alzheimer's disease. Clinical Neurophysiology, 122(12), 2355-2364. http://dx.doi.org /10.1016/j.clinph.2011.03.029

Babiloni, C., Del Percio, C., Capotosto, P., Noce, G., Infarinato, F., Muratori, C., ... Lupattelli, T. (2016). Cortical sources of resting state electroencephalographic rhythms differ in relapsing-remitting and secondary progressive multiple sclerosis. Clinical Neurophysiology, 127(1), 581-590. http://dx.doi.org/10.1016/j.clinph.2015.05.029
Barnden, L. R., Crouch, B., Kwiatek, R., Burnet, R., \& Del Fante, P. (2015). Evidence in chronic fatigue syndrome for severitydependent upregulation of prefrontal myelination that is independent of anxiety and depression. NMR in Biomedicine, 28(3), 404-413. http://dx.doi.org/10.1002/nbm.3261

Barnden, L. R., Crouch, B., Kwiatek, R., Burnet, R., Mernone, A., Chryssidis, S., ... Del Fante, P. (2011). A brain MRI study of chronic fatigue syndrome: Evidence of brainstem dysfunction and altered homeostasis. NMR in Biomedicine, 24(10), 13021312. http://dx.doi.org/10.1002/nbm.1692

Bassett, D. S., \& Bullmore, E. (2006). Small-world brain networks. The Neuroscientist, 12(6), 512-523. http://dx.doi.org/10.1177 $/ 1073858406293182$

Boissoneault, J., Letzen, J., Lai, S., O'Shea, A., Craggs, J., Robinson, M. E., \& Staud, R. (2016). Abnormal resting state functional connectivity in patients with chronic fatigue syndrome: An arterial spin-labeling fMRI study. Magnetic Resonance Imaging, 34(4), 603-608. http://dx.doi.org /10.1016/j.mri.2015.12.008

BrainMaster Discovery 24 [Apparatus]. (2011). Bedford, OH: BrainMaster Technologies, Inc.

Bullmore, E., \& Sporns, O. (2012). The economy of brain network organization. Nature Reviews Neuroscience, 13(5), 336-349. http://dx.doi.org/10.1038/nrn3214

Busichio, K., Tiersky, L. A., DeLuca, J., \& Natelson, B. H. (2004). Neuropsychological deficits in patients with chronic fatigue syndrome. Journal of the International Neuropsychological Society, 10(2), 278-285. http://dx.doi.org/10.1017 /s1355617704102178

Buzsáki, G. (2006). Rhythms of the Brain. New York, NY: Oxford University Press.

Buzsáki, G., \& Freeman, W. (2015). Editorial overview: Brain rhythms and dynamic coordination. Current Opinion in Neurobiology, 31, v-ix. http://dx.doi.org/10.1016 /j.conb.2015.01.016

Buzsáki, G., \& Watson, B. O. (2012). Brain rhythms and neural syntax: Implications for efficient coding of cognitive content and neuropsychiatric disease. Dialogues in Clinical Neuroscience, 14(4), 345-367.

Carruthers, B. M., Jain, A. K., De Meirleir, K. L., Peterson, D. L., Klimas, N. G., Lerner, A. M., ... van de Sande, M. I. (2003) Myalgic encephalomyelitits/chronic fatigue syndrome: Clinical working case definition, diagnostic and treatment protocols. Journal of Chronic Fatigue Syndrome, 11(1), 7-115. http://dx.doi.org/10.1300/J092v11n01_02

Carruthers, B. M., van de Sande, M. I., De Meirleir, K. L., Klimas, N. G., Broderick, G., Mitchell, T., ... Stevens, S. (2011). Myalgic encephalomyelitis: International Consensus Criteria. Journal of Internal Medicine, 270(4), 327-338. http://dx.doi.org/10.1111/j.1365-2796.2011.02428.x

Caseras, X., Mataix-Cols, D., Giampietro, V., Rimes, K. A., Brammer, M., Zelaya, F., ... Godfrey, E. L. (2006). Probing the working memory system in chronic fatigue syndrome: $A$ functional magnetic resonance imaging study using the $\mathrm{n}$ back task. Psychosomatic Medicine, 68(6), 947-955. http://dx.doi.org/10.1097/01.psy.0000242770.50979.5f

Castellanos, N. P., \& Makarov, V. A. (2006). Recovering EEG brain signals: Artifact suppression with wavelet enhanced independent component analysis. Journal of Neuroscience Methods, 158(2), 300-312. http://dx.doi.org/10.1016 /j.jneumeth.2006.05.033

Cockshell, S. J., \& Mathias, J. L. (2010). Cognitive functioning in chronic fatigue syndrome: A meta-analysis. Psychological Medicine, 40(8), 1253-1267. http://dx.doi.org/10.1017 /s0033291709992054

Constant, E. L., Adam, S., Gillain, B., Lambert, M., Masquelier, E., \& Seron, X. (2011). Cognitive deficits in patients with chronic fatigue syndrome compared to those with major depressive disorder and healthy controls. Clinical Neurology and 
Neurosurgery, 113(4), 295-302. http://dx.doi.org/10.1016 /j.clineuro.2010.12.002

Cook, D. B., O'Connor, P. J., Lange, G., \& Steffener, J. (2007). Functional neuroimaging correlates of mental fatigue induced by cognition among chronic fatigue syndrome patients and controls. Neurolmage, 36(1), 108-122. http://dx.doi.org /10.1016/j.neuroimage.2007.02.033

Crossley, N. A., Mechelli, A., Scott, J., Carletti, F., Fox, P. T., McGuire, P., \& Bullmore, E. T. (2014). The hubs of the human connectome are generally implicated in the anatomy of brain disorders. Brain, 137(8), 2382-2395. http://dx.doi.org/10.1093 /brain/awu132

Deco, G., Jirsa, V., \& Friston, K. J. (2012). The dynamical structural basis of brain activity. In M. I. Rabinovich, K. Friston, \& P. Varona (Eds.), Principles of Brain Dynamics: Global State Interactions (pp. 1-23). Cambridge, MA: MIT Press.

DeLuca, J., Johnson, S. K., \& Natelson, B. H. (1994). Neuropsychiatric status of patients with chronic fatigue syndrome: An overview. Toxicology and Industrial Health, 10(4-5), 513-522.

Dobbs, B. M., Dobbs, A. R., \& Kiss, I. (2001). Working memory deficits associated with chronic fatigue syndrome. Journal of the International Neuropsychological Society, 7(3), 285-293. http://dx.doi.org/10.1017/S1355617701733024

Flor-Henry, P., Lind, J. C., \& Koles, Z. J. (2010). EEG source analysis of chronic fatigue syndrome. Psychiatry Research, 181(2), 155-164. http://dx.doi.org/10.1016 /j.pscychresns.2009.10.007

Fukuda, K., Straus, S. E., Hickie, I., Sharpe, M. C., Dobbins, J. G., \& Komaroff, A. (1994). The chronic fatigue syndrome: A comprehensive approach to its definition and study. Annals of Internal Medicine, 121(12), 953-959.

Gay, C. W., Robinson, M. E., Lai, S., O'Shea, A., Craggs, J. G., Price, D. D., \& Staud, R. (2016). Abnormal resting-state functional connectivity in patients with chronic fatigue syndrome: Results of seed and data-driven analyses. Brain Connectivity, 6(1), 48-56. http://dx.doi.org/10.1089 /brain.2015.0366

Gloor, P., Ball, G., \& Schaul, N. (1977). Brain lesions that produce delta waves in the EEG. Neurology, 27(4), 326-333. http://dx. doi.org/10.1212/WNL.27.4.326

Grafman, J., Schwartz, V., Dale, J. K., Scheffers, M., Houser, C., \& Straus, S. E. (1993). Analysis of neuropsychological functioning in patients with chronic fatigue syndrome. Journal of Neurology, Neurosurgery, \& Psychiatry, 56(6), 684-689.

Grech, R., Cassar, T., Muscat, J., Camilleri, K. P., Fabri, S. G., Zervakis, M., ... Vanrumste, B. (2008). Review on solving the inverse problem in EEG source analysis. Journal of NeuroEngineering and Rehabilitation, 5, 25. http://dx.doi.org /10.1186/1743-0003-5-25

Harris, P. A., Taylor, R., Thielke, R., Payne, J., Gonzalez, N., \& Conde, J. G. (2009). Research electronic data capture (REDCap)—A metadata-driven methodology and workflow process for providing translational research informatics support. Journal of Biomedical Informatics, 42(2), 377-381. http://dx.doi.org/10.1016/j.jbi.2008.08.010

Hata, M., Kazui, H., Tanaka, T., Ishii, R., Canuet, L., PascualMarqui, R. D., ... Takeda, M. (2016). Functional connectivity assessed by resting state EEG correlates with cognitive decline of Alzheimer's disease: An eLORETA study. Clinical Neurophysiology, 127(2), 1269-1278. http://dx.doi.org /10.1016/j.clinph.2015.10.030

Hopkins, R. O., \& Jackson, J. C. (2006). Long-term neurocognitive function after critical illness. Chest, 130(3), 869-878. http://dx.doi.org/10.1378/chest.130.3.869

Humphries, M. D., \& Gurney, K. (2008). Network 'small-worldness': A quantitative method for determining canonical network equivalence. PloS ONE, 3(4), e0002051. http://dx.doi.org/10.1371/journal.pone.0002051
Jason, L. A., So, S., Brown, A. A., Sunnquist, M., \& Evans, M. (2015). Test-retest reliability of the DePaul Symptom Questionnaire. Fatigue Biomedicine Health \& Behavior, 3(1), 16-32. http://dx.doi.org/10.1080/21641846.2014.978110

Jason, L. A., Sunnquist, M., Brown, A., Furst, J., Cid, M., Farietta, J., ... Strand, E. B. (2015). Factor analysis of the DePaul Symptom Questionnaire: Identifying core domains. Journal of Neurology and Neurobiology, 1(4). http://dx.doi.org/10.16966 /2379-7150.114

Jason, L. A., Zinn, M. L., \& Zinn, M. A. (2015). Myalgic encephalomyelitis: Symptoms and biomarkers. Current Neuropharmacology, 13(5), 701-734. http://dx.doi.org /10.2174/1570159X13666150928105725

John, E. R. (2005). From synchronous neuronal discharges to subjective awareness? In S. Laureys (Ed.), Progress in Brain Research (Vol. 150, pp. 55-68). London, England: Elsevier.

Johnson, S. K., DeLuca, J., \& Natelson, B. H. (1996). Assessing somatization disorder in the chronic fatigue syndrome. Psychosomatic Medicine, 58(1), 50-57. http://dx.doi.org /10.1097/00006842-199601000-00008

Jurcak, V., Tsuzuki, D., \& Dan, I. (2007). 10/20, 10/10, and 10/5 systems revisited: Their validity as relative head-surfacebased positioning systems. Neurolmage, 34(4), 1600-1611. http://dx.doi.org/10.1016/j.neuroimage.2006.09.024

Kierkels, J. J. M., van Boxtel, G. J. M., \& Vogten, L. L. M. (2006) A model-based objective evaluation of eye movement correction in EEG recordings. IEEE Transactions on Biomedical Engineering, 53(2), 246-253. http://dx.doi.org /10.1109/tbme.2005.862533

Kim, B.-H., Namkoong, K., Kim, J.-J., Lee, S., Yoon, K. J., Choi, M., \& Jung, Y.-C. (2015). Altered resting-state functional connectivity in women with chronic fatigue syndrome. Psychiatry Research, 234(3), 292-297. http://dx.doi.org /10.1016/j.pscychresns.2015.10.014

Kirk, R. E. (2013). Experimental design: Procedures for the behavioral sciences (4th ed.). Los Angeles, CA: SAGE Publications.

Klimesch, W., Freunberger, R., Sauseng, P., \& Gruber, W. (2008). A short review of slow phase synchronization and memory: Evidence for control processes in different memory systems? Brain Research, 1235, 31-44. http://dx.doi.org /10.1016/j.brainres.2008.06.049

Komaroff, A. L., \& Buchwald, D. (1991). Symptoms and signs of chronic fatigue syndrome. Reviews of Infectious Diseases, 13(Suppl. 1), S8-S11. http://dx.doi.org/10.1093/clinids 113.Supplement_1.S8

Koziol, L. F., \& Budding, D. E. (2009). Subcortical structures and cognition: Implications for neuropsychological assessment. New York, NY: Springer.

Kruschwitz, J. D., List, D., Waller, L., Rubinov, M., \& Walter, H. (2015). GraphVar: A user-friendly toolbox for comprehensive graph analyses of functional brain connectivity. Journal of Neuroscience Methods, 245, 107-115. http://dx.doi.org /10.1016/j.jneumeth.2015.02.021

Lange, G., Steffener, J., Cook, D. B., Bly, B. M., Christodoulou, C., Liu, W.-C., ... Natelson, B. H. (2005). Objective evidence of cognitive complaints in Chronic Fatigue Syndrome: A BOLD fMRI study of verbal working memory. Neurolmage, 26(2), 513-524. http://dx.doi.org/10.1016 /j.neuroimage.2005.02.011

Le Van Quyen, M. (2011). The brainweb of cross-scale interactions. New Ideas in Psychology, 29(2), 57-63. http://dx.doi.org/10.1016/j.newideapsych.2010.11.001

Lehmann, D., Faber, P. L., Gianotti, L. R. R., Kochi, K., \& Pascual-Marqui, R. D. (2006). Coherence and phase locking in the scalp EEG and between LORETA model sources, and microstates as putative mechanisms of brain temporo-spatia functional organization. Journal of Physiology-Paris, 99(1), 29-36. http://dx.doi.org/10.1016/j.jphysparis.2005.06.005 
Majer, M., Welberg, L. A., Capuron, L., Miller, A. H., Pagnoni, G., \& Reeves, W. C. (2008). Neuropsychological performance in persons with chronic fatigue syndrome: Results from a population-based study. Psychosomatic Medicine, 70(7), 829-836. http://dx.doi.org/10.1097/PSY.0b013e31817b9793

Menon, V. (2011). Large-scale brain networks and psychopathology: A unifying triple network model. Trends in Cognitive Sciences, 15(10), 483-506. http://dx.doi.org /10.1016/j.tics.2011.08.003

Menon, V. (2012). Functional connectivity, neurocognitive networks, and brain dynamics. In M. I. Rabinovich, K. J. Friston, \& P. Varona (Eds.), Principles of Brain Dynamics: Global State Interactions (pp. 27-47). Cambridge, MA: MIT Press.

Minati, L., Varotto, G., D'Incerti, L., Panzica, F., \& Chan, D. (2013). From brain topography to brain topology: Relevance of graph theory to functional neuroscience. Neuroreport, 24(10), $\quad$ 536-543. http://dx.doi.org/10.1097 MNR.0b013e3283621234

Murdock, K. W., Wang, X. S., Shi, Q., Cleeland, C. S., Fagundes, C. P., \& Vernon, S. D. (2016). The utility of patient-reported outcome measures among patients with myalgic encephalomyelitis/chronic fatigue syndrome. Quality of Life Research, 26(4), 913-921. http://dx.doi.org/10.1007/s11136016-1406-3

Nakatomi, Y., Mizuno, K., Ishii, R., Wada, Y., Tanaka, M., Tazawa, S., ... Watanabe, Y. (2014). Neuroinflammation in patients with Chronic Fatigue Syndrome/Myalgic Encephalomyelitis: An ${ }^{11} \mathrm{C}-(\mathrm{R})-\mathrm{PK} 11195$ PET study. Journal of Nuclear Medicine, 55(6), 945-950. http://dx.doi.org /10.2967/jnumed.113.131045

Neuroguide (Version 2.8.7) [Computer software]. (2016). St. Petersburg, FL: Applied Neuroscience, Inc.

Niedermeyer, E., \& Lopes da Silva, F. H. (2005). Electroencephalography: Basic principles, clinical applications and related fields (5th ed.). Philadelphia, PA: Lippincott Williams and Wilkins.

Nunez, P. L., Srinivasan, R., \& Fields, R. D. (2015). EEG functional connectivity, axon delays and white matter disease. Clinical Neurophysiology, 126(1), 110-120. http://dx.doi.org /10.1016/j.clinph.2014.04.003

Ocon, A. J. (2013). Caught in the thickness of brain fog: Exploring the cognitive symptoms of Chronic Fatigue Syndrome. Frontiers in Physiology, 4, 63. http://dx.doi.org/10.3389 /fphys.2013.00063

Pascual-Marqui, R. D. (2007a). Coherence and phase synchronization: Generalization to pairs of multivariate time series, and removal of zero-lag contribution (arXiv:0706.1776v3 [stat.ME]). Retrieved from http://arxiv.org /pdf/0706.1776

Pascual-Marqui, R. D. (2007b). Discrete, 3D distributed linear imaging methods of electric neuronal activity. Part 1: Exact, zero error localization (arXiv:0710.3341 [math-ph]). Retrieved from https://arxiv.org/ftp/arxiv/papers/0710/0710.3341.pdf

Pascual-Marqui, R. D. (2007c). Instantaneous and lagged measurements of linear and nonlinear dependence between groups of multivariate time series: Frequency decomposition (arXiv:0711.1455[stat.ME]). Retrieved from https://arxiv.org /pdf/0711.1455v1

Pascual-Marqui, R. D. (2015). LORETA-KEY software (Version 2015-12-22). Zurich, Switzerland: KEY Institute for Brain-Mind Research. Retrieved from http://www.uzh.ch/keyinst /loreta.htm

Pascual-Marqui, R. D., Lehmann, D., Koukkou, M., Kochi, K., Anderer, P., Saletu, B., ... Kinoshita, T. (2011). Assessing interactions in the brain with exact low-resolution electromagnetic tomography. Philosophical Transactions of the Royal Society A, Mathematical, Physical, and Engineering Sciences, 369(1952), 3768-3784. http://dx.doi.org/10.1098 /rsta.2011.0081
Puri, B. K., Jakeman, P. M., Agour, M., Gunatilake, K. D. R., Fernando, K. A. C., Gurusinghe, A. I., ... Gishen, P. (2012). Regional grey and white matter volumetric changes in myalgic encephalomyelitis (chronic fatigue syndrome): A voxel-based morphometry 3 T MRI study. British Journal of Radiology, 85(1015), e270-e273. http://dx.doi.org/10.1259/bjr 193889091

Rossini, P. M., Rossi, S., Babiloni, C., \& Polich, J. (2007). Clinical neurophysiology of aging brain: From normal aging to neurodegeneration. Progress in Neurobiology, 83(6), 375400. http://dx.doi.org/10.1016/j.pneurobio.2007.07.010

Rubinov, M., \& Sporns, O. (2010). Complex network measures of brain connectivity: Uses and interpretations. Neurolmage, 52(3), 1059-1069. http://dx.doi.org/10.1016 /j.neuroimage.2009.10.003

Sauseng, P., \& Klimesch, W. (2008). What does phase information of oscillatory brain activity tell us about cognitive processes? Neuroscience \& Biobehavioral Reviews, 32(5), 1001-1013. http://dx.doi.org/10.1016/j.neubiorev.2008.03.014

Schaul, N., Gloor, P., \& Gotman, J. (1981). The EEG in deep midline lesions. Neurology, 31(2), 157-167. http://dx.doi.org/ 10.1212/WNL.31.2.157

Sepulcre, J. (2014). Functional streams and cortical integration in the human brain. The Neuroscientist, 20(5), 499-508. http://dx.doi.org/10.1177/1073858414531657

Sherlin, L., Budzynski, T., Kogan Budzynski, H., Congedo, M., Fischer, M. E., \& Buchwald, D. (2007). Low-resolution electromagnetic brain tomography (LORETA) of monozygotic twins discordant for chronic fatigue syndrome. Neurolmage, 34(4), 1438-1442. $\quad \mathrm{http}: / / \mathrm{dx}$. doi.org/10.1016 /j.neuroimage.2006.11.007

Sporns. (2013). Structure and function of complex brain networks. Dialogues in Clinical Neuroscience, 15(3), 247-262.

Sporns, O., \& Honey, C. J. (2006). Small worlds inside big brains. Proceedings of the National Academy of Sciences, 103(51), 19219-19220. http://dx.doi.org/10.1073/pnas.0609523103

Stam, C. J. (2010). Characterization of anatomical and functional connectivity in the brain: A complex networks perspective. International Journal of Psychophysiology, 77(3), 186-194. http://dx.doi.org/10.1016/j.ijpsycho.2010.06.024

Stam, C. J. (2014). Modern network science of neurological disorders. Nature Reviews Neuroscience, 15(10), 683-695. http://dx.doi.org/10.1038/nrn3801

Steriade, M. (2005). Cellular substrates of brain rhythms. In E. Niedermeyer \& F. H. Lopes de Silva (Eds.), Electroencephalography: Basic principles, clinical applications and related fields (5th ed., pp. 31-83). Philadelphia, PA: Lippincott Williams and Wilkins.

Steriade, M., \& Paré, D. (2007). Gating in cerebral networks. New York, NY: Cambridge University Press.

Telesford, Q. K., Simpson, S. L., Burdette, J. H., Hayasaka, S., \& Laurienti, P. J. (2011). The brain as a complex system: Using network science as a tool for understanding the brain. Brain Connectivity, 1(4), 295-308. http://dx.doi.org/10.1089 /brain.2011.0055

Thatcher, R. W. (2016). Handbook of Quantitative Electroencephalography and EEG Biofeedback. St Petersburg, FL: ANI Publishing.

Thatcher, R. W., North, D. M., \& Biver, C. J. (2008). Intelligence and EEG phase reset: A two compartmental model of phase shift and lock. Neurolmage, 42(4), 1639-1653. http://dx.doi.org/10.1016/j.neuroimage.2008.06.009

Thomas, M., \& Smith, A. (2009). An investigation into the cognitive deficits associated with chronic fatigue syndrome. The Open Neurology Journal, 3, 13-23. http://dx.doi.org $/ 10.2174 / 1874205 \times 00903010013$

Van Den Eede, F., Moorkens, G., Hulstijn, W., Maas, Y., Schrijvers, D., Stevens, S. R., ... Sabbe, B. G. C. (2011). Psychomotor function and response inhibition in chronic 
fatigue syndrome. Psychiatry Research, 186(2-3), 367-372. http://dx.doi.org/10.1016/j.psychres.2010.07.022

van den Heuvel, M. P., \& Sporns, O. (2013). An anatomical substrate for integration among functional networks in human cortex. The Journal of Neuroscience, 33(36), 14489-14500. http://dx.doi.org/10.1523/jneurosci.2128-13.2013

van Straaten, E. C. W., \& Stam, C. J. (2013). Structure out of chaos: Functional brain network analysis with EEG, MEG, and functional MRI. European Neuropsychopharmacology, 23(1), $\quad 7-18 . \quad$ http://dx.doi.org/10.1016 /j.euroneuro.2012.10.010

Vecchio, F., Miraglia, F., Curcio, G., Altavilla, R., Scrascia, F., Giambattistelli, F., ... Rossini, P. M. (2015). Cortical brain connectivity evaluated by graph theory in dementia: A correlation study between functional and structural data. Journal of Alzheimer's Disease, 45(3), 745-756. http://dx.doi.org/10.3233/jad-142484

Vecchio, F., Miraglia, F., Curcio, G., Della Marca, G., Vollono, C., Mazzucchi, E., ... Rossini, P. M. (2015). Cortical connectivity in fronto-temporal focal epilepsy from EEG analysis: A study via graph theory. Clinical Neurophysiology, 126(6), 11081116. http://dx.doi.org/10.1016/j.clinph.2014.09.019

Vecchio, F., Miraglia, F., Porcaro, C., Cottone, C., Cancelli, A., Rossini, P. M., \& Tecchio, F. (2017). Electroencephalography-Derived Sensory and Motor Network Topology in Multiple Sclerosis Fatigue. Neurorehabilitation and Neural Repair, 31(1), 56-64. http://dx.doi.org/10.1177 /1545968316656055

Vecchio, F., Miraglia, F., Quaranta, D., Granata, G., Romanello, R., Marra, C., ... Rossini, P. M. (2016). Cortical connectivity and memory performance in cognitive decline: A study via graph theory from EEG data. Neuroscience, 316, 143-150. http://dx.doi.org/10.1016/j.neuroscience.2015.12.036

Vysata, O., Kukal, J., Prochazka, A., Pazdera, L., Simko, J., \& Valis, M. (2014). Age-related changes in EEG coherence. Neurologia i Neurochirurgia Polska, 48(1), 35-38. http://dx.doi.org/10.1016/j.pjnns.2013.09.001

Wallstrom, G. L., Kass, R. E., Miller, A., Cohn, J. F., \& Fox, N. A. (2004). Automatic correction of ocular artifacts in the EEG: A comparison of regression-based and component-based methods. International Journal of Psychophysiology, 53(2), 105-119. http://dx.doi.org/10.1016/j.ijpsycho.2004.03.007

Watts, D. J., \& Strogatz, S. H. (1998). Collective dynamics of "small-world" networks. Nature, 393(6684), 440-442. http://dx.doi.org/10.1038/30918

Westmoreland, B. (2005). The EEG in Cerebral Inflammatory Processes. In E. Niedermeyer \& F. H. Lopes da Silva (Eds.), Electroencephalography: Basic principles, clinical applications and related fields (5th ed., pp. 323-337). Philadelphia, PA: Lippincott Williams and Wilkins.

Wig, G. S., Schlaggar, B. L., \& Petersen, S. E. (2011). Concepts and principles in the analysis of brain networks. Annals of the New York Academy of Sciences, 1224, 126-146. http://dx.doi.org/10.1111/j.1749-6632.2010.05947.x

Wortinger, L. A., Endestad, T., Melinder, A. M. D., Øie, M. G., Sevenius, A., \& Bruun Wyller, V. (2016). Aberrant restingstate functional connectivity in the salience network of adolescent chronic fatigue syndrome. PLOS ONE, 11(7), e0159351. http://dx.doi.org/10.1371/journal.pone.0159351

Zinn, M. A., Zinn, M. L., Norris, J. L., Valencia, I., Montoya, J. G., \& Maldonado, J. R. (2014). Cortical hypoactivation during resting EEG suggests central nervous system pathology in patients with Chronic Fatigue Syndrome. Paper presented at the Symposium conducted at the meeting of IACFS/ME 2014 Biennial Conference, San Francisco, CA, USA.

Zinn, M. L., Zinn, M. A., \& Jason, L. A. (2016). Intrinsic functional hypoconnectivity in core neurocognitive networks suggests central nervous system pathology in patients with myalgic encephalomyelitis: A pilot study. Applied Psychophysiology and Biofeedback, 41(3), 283-300. http://dx.doi.org/10.1007 /s10484-016-9331-3

Received: August 18, 2017

Accepted: August 30, 2017

Published: December 8, 2017 